\title{
Motor Skill Learning Depends on Protein Synthesis in Motor Cortex after Training
}

\author{
Andreas R. Luft, ${ }^{*}$ Manuel M. Buitrago, ${ }^{*}$ Thomas Ringer, Johannes Dichgans, and Jörg B. Schulz \\ Department of General Neurology and Hertie Institute for Clinical Brain Research, University of Tübingen, 72076 Tübingen, Germany
}

The role of protein synthesis in memory consolidation is well established for hippocampus-dependent learning and synaptic plasticity. Whether protein synthesis is required for motor skill learning is unknown. We hypothesized that skill learning is interrupted by protein synthesis inhibition (PSI). We intended to test whether local protein synthesis in motor cortex or cerebellum is required during skill acquisition and consolidation. Anisomycin (ANI; $100 \mu \mathrm{g} / \mu \mathrm{l}$ in $1 \mu \mathrm{l}$ of PBS) injected into motor cortex, posterior parietal cortex, or cerebellum produced $84.0 \pm 1.44 \%$ (mean \pm SEM), $85.9 \pm 2.31 \%$, and $87.3 \pm 0.17 \%$ of PSI 60 min after administration, respectively. In motor cortex, protein synthesis was still reduced at $24 \mathrm{hr}(72.0 \pm 4.68 \% \mathrm{PSI})$ but normalized at $48 \mathrm{hr}$ after a second injection given $24 \mathrm{hr}$ after the first. To test for the effects of PSI on learning of a skilled reaching task, ANI was injected into motor cortex contralateral to the trained limb or into ipsilateral cerebellum immediately after daily training sessions 1 and 2 . Two control groups received motor cortex injections of vehicle or ANI injections into contralateral parietal cortex. Control and cerebellar animals showed a sigmoid learning curve, which plateaued after day 4. PSI in motor cortex significantly reduced learning during days 1-4. Thereafter, when protein synthesis normalized, learning was reinitiated. ANI injections into motor cortex did not induce a motor deficit, because animals injected during the performance plateau did not deteriorate. This demonstrates that motor skill learning depends on de novo synthesis of proteins in motor cortex after training.

Key words: motor skill learning; memory; protein synthesis; anisomycin; rat; cerebellum

\section{Introduction}

Motor skill learning is essential for development and for environmental adaptations during adult life. Neuroplasticity in motor cortices has been shown to play a role in motor learning (Li et al., 2001). Such plasticity may also mediate recovery after brain lesions (Hallett, 2001). Although motor skill learning seems independent of hippocampal circuits (Gould et al., 2002; Davey et al., 2003), sensorimotor cortex and cerebellum are likely involved (Hikosaka et al., 2002). However, the contribution of each of these brain regions to acquisition and storage of a motor skill is unknown.

The role of protein synthesis has long been established for hippocampus-dependent learning and memory. Reviewing the experimental evidence, Davis and Squire (1984) concluded that (1) inhibition of cerebral protein synthesis (PSI) does not interfere with behavior, (2) acquisition under PSI is normal for brief training, but (3) the retention of learned content is impaired, and (4) depending on the paradigm, initiation of PSI after training

Received Dec. 24, 2003; revised June 6, 2004; accepted June 6, 2004.

This work was supported by a grant from the Deutsche Forschungsgemeinschaft (Lu 748/3). We thank Prof. Larry Squire (University of California, San Diego, School of Medicine, San Diego, CA), Prof. Hans-Christian Pape (University of Magdeburg, Magdeburg, Germany), and Prof. Charles Nicholson (New York University, New York, NY) for their valuable advice and discussion.

${ }^{*}$ A.R.L. and M.M.B. contributed equally to this work.

Correspondence should be addressed to Dr. Andreas Luft, Department of General Neurology, Hertie Institute for Clinical Brain Research, University of Tübingen, Hoppe-Seyler-Strasse 3, 72076 Tübingen, Germany. E-mail: aluft@anatom.uni-tuebingen.de.

DOI:10.1523/JNEUROSCI.1034-04.2004

Copyright $\odot 2004$ Society for Neuroscience $\quad$ 0270-6474/04/246515-06\$15.00/0 produces amnesia, but this amnesia is generally not as profound as the one obtained by inducing PSI before training. Recently, PSI in the nucleus accumbens has been shown to inhibit consolidation of instrumental learning (Hernandez et al., 2002). Hence, establishing persistent memory traces seems to require de novo synthesized proteins (Nader, 2003). These proteins may be used to reorganize the synaptic or dendritic structure (Steward and Schuman, 2001). Alternatively, proteins themselves have been suggested as carriers of memory content (Arshavsky, 2003).

Whether protein synthesis is required for motor skill learning has not been demonstrated yet. Here, we show in a rat paradigm of learning of a precision forelimb reaching movement that skill learning over a period of several days depends on de novo synthesis of proteins in motor cortex after training.

\section{Materials and Methods}

Animals and protocols. Sixty adult male Long-Evans rats (8 weeks of age; raised within our stock) were used in four experiments. First, the level of PSI after an intracortical injection of anisomycin (ANI) was assessed at three different time points in motor cortex and at one time point in parietal cortex and cerebellum ( $n=3$ in each group). Second, the spread of the injected volume of anisomycin in motor cortex, parietal cortex, and cerebellum was simulated by injections of India ink (five rats per site) (Berman and Dudai, 2001). Third, motor skill learning was evaluated after inducing PSI in contralateral motor cortex or in ipsilateral cerebellum (relative to the trained forelimb) by injection of ANI immediately after training sessions (days) 1 and 2. At identical time points, control groups received vehicle injections into motor cortex or ANI injections into contralateral parietal cortex ( $n=6$ per group). Skill training was continued until session 8 . The investigator training the animals (M.M.B.) 
was blinded to group assignment. Finally, to test for possible motor deficits caused by local ANI injections into motor cortex, the drug was injected after training animals for $11 \mathrm{~d}$ when they had long reached plateau performance $(n=6)$. Injections were performed immediately after sessions 11 and 12, and training was continued until session 16. The brains of three of these animals were evaluated histologically to screen for cortical injury.

Rats were kept in a $12 \mathrm{hr}$ day/night cycle with ad libitum access to water. They were housed individually. Training was performed at the beginning of the dark phase of the cycle. Body weight was recorded daily. All procedures were approved by the Animal Care and Use Committee of the University of Tübingen and the accountable State Agency.

Motor skill training. Rats were trained to reach and grasp for a food pellet (45 mg; Bioserve, Frenchtown, NJ) placed outside the cage. The pellet rested on a cylindrical pedestal with $7 \mathrm{~mm}$ diameter. The pellet could be accessed through a $1 \times 5 \mathrm{~cm}$ vertical window in the cage wall. The window was covered by a motorized sliding door, which could be opened by the rat by poking its nose to an inductive sensor in the opposite wall of the cage. The door closed automatically $1 \mathrm{sec}$ after the pellet was removed from the pedestal (inductive sensor built into the pedestal).

Before training, animals were food deprived for $24 \mathrm{hr}$. Afterward, they received a restricted diet of $\sim 50 \mathrm{gm} / \mathrm{kg}$ body weight adjusted to keep their weight constant. Training began by familiarizing the rat with the cage and the food pellets for $1 \mathrm{hr}$. During the next $4 \mathrm{~d}$ (sessions -3 to 0 ), rats were pretrained with the pellet in close proximity to the window (window-to-pellet distance, $<5 \mathrm{~mm}$ ). From this position, the pellet could be retrieved with the tongue. During pretraining, animals learned to open the door. Surgical preparations (see below) were performed after pretraining. Three days of recovery were allowed after surgery.

Motor skill learning was initiated by increasing the window-to-pellet distance to $1.5 \mathrm{~cm}$, requiring the rat to use the forelimb to retrieve the pellet. Each animal quickly (during the first 5-10 reaches) demonstrated a forelimb preference. The pedestal was then shifted from the midwindow position $1 \mathrm{~cm}$ to either side (right side if left forelimb was preferred and vice versa). The animals thereby retained their forelimb preference for the entire training period. No rat was found to use the nonpreferred forelimb at any time. During each training session, rats were allowed to access 100 pellets (100 door openings in each session). A trial ended when the rat removed the pellet from the pedestal (by grasping or pushing). This could involve several reaching attempts. The session duration decreased from $33.4 \pm 12.3$ min $($ mean \pm SD) in session 1 to $21.3 \pm 10.3$ min in session 8 .

Latencies between pellet removal and subsequent door opening were recorded as a measure of learning the concept of door opening. To measure the improvement of precision reaching, the number of successful reaches (pellet grasped, held in the paw, and pulled back from the pedestal) was counted and divided by the total number of trials (100).

Intracortical injections. Injection cannula systems (Unimed, Lausanne, Switzerland) were implanted after pretraining, and $3 \mathrm{~d}$ of recovery were allowed after surgery. The system consisted of an occlusion obturator and external guide cannula (diameter, $400 \mu \mathrm{m}$ ), through which a fine needle ( 35 gauge; Hamilton, Martinsried, Germany) was advanced at the time of injection. For implantation, animals were anesthetized with ketamine ( $10 \%$, i.p., $70-100 \mathrm{mg} / \mathrm{kg}$ body weight) and xylazine (2\%, i.p., $5-10 \mathrm{mg} / \mathrm{kg}$ body weight). Two guide cannulas were implanted through burrholes (1.5 mm diameter) into bilateral motor cortices (coordinates relative to bregma, $1 \mathrm{~mm}$ anterior, $3 \mathrm{~mm}$ lateral; depth, $900 \mu \mathrm{m}$ ), posterior parietal cortices ( $6 \mathrm{~mm}$ posterior, $3 \mathrm{~mm}$ lateral; depth, $900 \mu \mathrm{m}$ ), or anterior lobe of the cerebellar hemispheres $(3 \mathrm{~mm}$ posterior to the interaural line, $3 \mathrm{~mm}$ lateral; depth, $600 \mu \mathrm{m}$ ). The coordinates of motor cortex implants were selected on the basis of published somatotopy data (Neafsey et al., 1986). The site of cerebellar implants was chosen with respect to the somatotopical organization of the cerebellum (Atkins and Apps, 1997). The zone receiving predominantly peripheral input from the ipsilateral forelimb via climbing fibers is located in the paramedian lobule (C1) and projects to the coordinates $-3 \mathrm{~mm}$ posterior of the inter-aural line and $2.8 \mathrm{~mm}$ lateral of the midline (Paxinos and Watson, 1998). Immediately lateral of $\mathrm{C} 1$ is zone $\mathrm{C} 2$, receiving input from both forelimbs; lateral of C2 is C3 with, again, ipsilateral input. Zones C2 and C3 have a smaller width in mediolateral direction than $\mathrm{C} 1$. Therefore, implants were placed $3 \mathrm{~mm}$ lateral of the midline. These receptive fields defined by climbing fiber input are largely congruent with mossy fiber receptive fields (Brown and Bower, 2001).

Bilateral implantation of the cannula was necessary, because at the time of implantation (after pretraining), the preferred forelimb was not yet defined. We chose not to determine forelimb preference before surgery to avoid the interruption of the motor learning process by anesthesia, surgical manipulations, and the recovery period. Burrholes and cannulas were covered with bone cement. The bone cement entrenched a small screw anchored in the occipital bone, which ensured firm implant attachment in the freely moving rat.

Immediately after motor skill training sessions 1 and 2, rats were sedated briefly with ether (duration, $<2 \mathrm{~min}$ ) and injected with $100 \mu \mathrm{g}$ of ANI dissolved in $1 \mu \mathrm{l}$ of PBS or with PBS alone (5 $\mu \mathrm{l}$ microsyringe; Hamilton) into the motor cortex contralateral to the preferred forelimb. ANI injections into parietal cortex were also performed contralaterally, whereas in cerebellum, ANI (same dose) was applied to the ipsilateral hemisphere. Injection speed was $1 \mu \mathrm{l} / \mathrm{min}$, and one additional minute with the injection needle in place was allowed for diffusion (Rosenblum et al., 1993; Kleim et al., 2003). The time elapsed between the last reach, and successful injection was carefully monitored and was shorter than 5 min for all cases. We a priori chose to give two injections (after sessions 1 and 2) to increase potential effects of PSI on motor learning.

Evaluation of PSI. The level of PSI after application of ANI was measured via incorporation of ${ }^{35} \mathrm{~S}$-labeled methionine (Amersham Biosciences, Freiburg, Germany) into newly synthesized proteins. In motor cortex, the levels of PSI were evaluated at different time points: $60 \mathrm{~min}$ after the first ANI injection, $24 \mathrm{hr}$ after the first injection, and $48 \mathrm{hr}$ after the second application. In parietal cortex and cerebellum, PSI was measured at $60 \mathrm{~min}$ after one ANI injection. All injections of ANI were unilateral, using the above described cannula system. Vehicle (PBS) was given to the hemisphere contralateral to the ANI injection. Thirty minutes after ANI or vehicle, ${ }^{35} \mathrm{~S}$-methionine $(2 \mu \mathrm{l}$ of $10 \mu \mathrm{Ci} / \mu \mathrm{l} ; 1 \mu \mathrm{l} / \mathrm{min})$ was injected bilaterally. Thirty minutes after methionine injection, animals were killed with an overdose of pentobarbital, and the target regions (motor cortex, parietal cortex, and cerebellum) were dissected bilaterally. Tissue blocks included the entire motor cortex $(4 \mathrm{~mm}$ in mediolateral width and $5 \mathrm{~mm}$ in length, with the injection coordinate as center), a region of parietal cortex of comparable size, and the entire cerebellar hemisphere. Tissue samples were weighed and homogenized in $1.5 \mathrm{ml}$ of lysis buffer per $100 \mathrm{mg}$ of tissue (1\% SDS, $62 \mathrm{~mm}$ Tris, pH 7.4, $40 \mathrm{~mm}$ DTT, $62 \mathrm{~mm}$ imidazol, $10 \%$ glycerol). The homogenate was boiled for 5 $\min$ at $95^{\circ} \mathrm{C}$ and centrifuged $\left(10,000 \mathrm{rpm}\right.$ for $15 \mathrm{~min}$ at $\left.4^{\circ} \mathrm{C}\right)$. Protein precipitation was achieved by mixing the supernatant with TCA $(25 \%$ final concentration) followed by incubation for $60 \mathrm{~min}$ (ice cold) and centrifugation $\left(13,000 \mathrm{rpm}\right.$ for $20 \mathrm{~min}$ at $\left.4^{\circ} \mathrm{C}\right)$. A scintillation counter was used to measure radioactivity of the protein pellet and of the supernatant. For each animal, a pellet-to-supernatant index, $i$, was computed as the ratio of pellet scintillation counts to supernatant counts. The percentage of protein synthesis inhibition was then calculated using the following formula: $\left[1-i_{\text {ANI-injected side }} /\right.$ mean $\left.\left(i_{\text {PBS-injected side }}\right)\right] * 100$, with mean $\left(i_{\text {PBS-injected hemisphere }}\right)$ referring to the mean of all three PBSinjected hemispheres within one group.

Evaluation of ANI spread and diffusion. The spread of the injected volume of ANI was estimated by injecting India ink as a visible simulant in five rats per injection site (cerebellum, motor cortex, or parietal cortex), as performed by Berman and Dudai (2001). One microliter of India ink was injected via the implanted cannula system using identical injection techniques as used for ANI. One hour after the injection, animals were anesthetized (pentobarbital $100 \mathrm{mg} / \mathrm{kg}$ ) and perfused with $4 \%$ paraformaldehyde (PFA) in PBS, and the brains were removed, postfixed for $1 \mathrm{~d}$, cryoprotected, and frozen. A cryostat was used to reach the injection site in a coronal plane. The spread of the injected volume was assessed by measuring horizontal and vertical diameters of the ink blot at the level of the cannula tract.

The spread of the injected volume is driven by injection pressure. Subsequently, diffusion occurs. Diffusion of ANI was estimated according to Nicholson's (1985) Equation 13. 


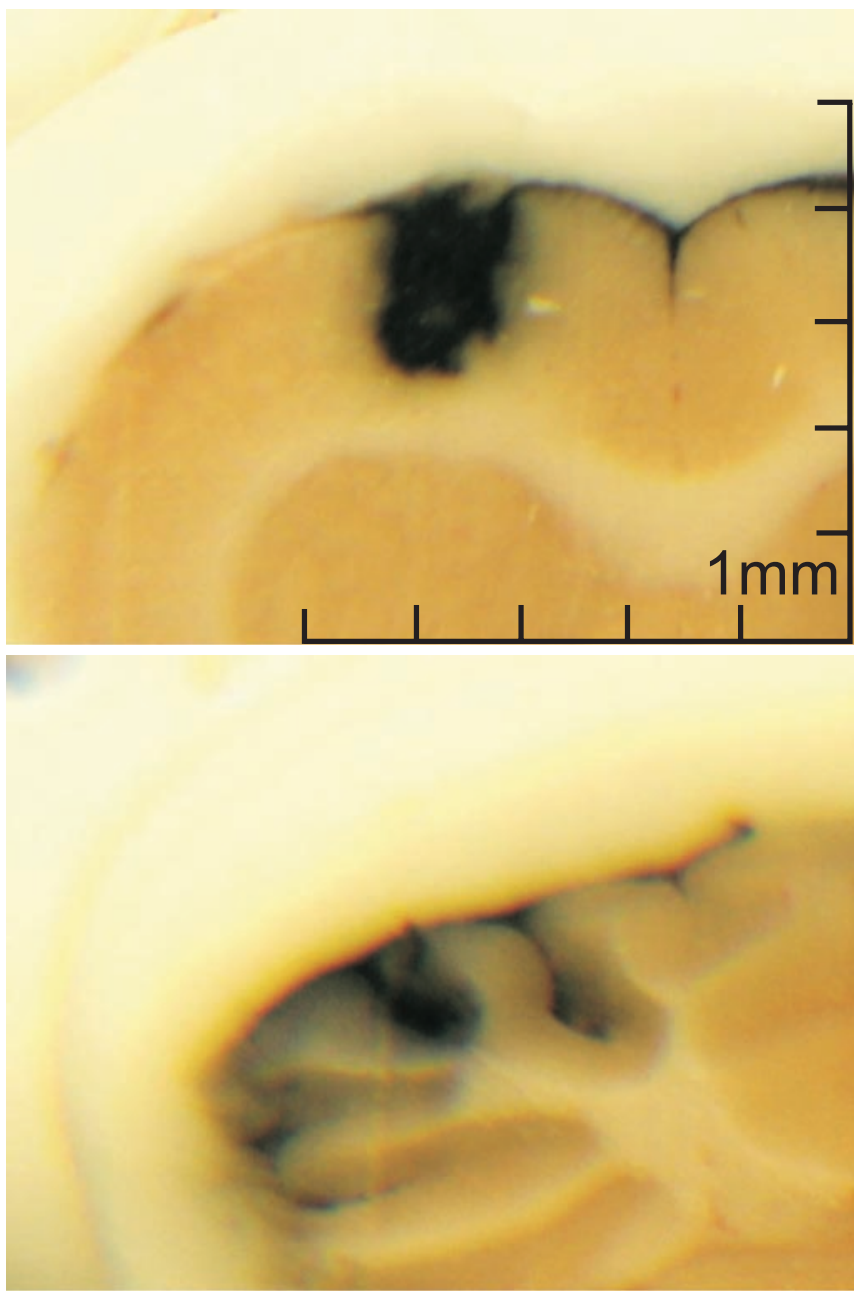

Figure 1. Spread of ink injected into motor cortex and cerebellum (experiment 2). India ink was used as a visual stimulant to estimate the initial spread of ANI in cortex (top) and in cerebellum (bottom). Sections from one exemplary animal are shown.

Histology. Histological assessment of cortical integrity was performed in three animals trained for 16 sessions and injected with ANI into motor cortex after sessions 11 and 12. Animals were deeply anesthetized with pentobarbital and transcardially perfused with $4 \%$ PFA in PBS. The brain was extracted, cryoprotected in $20 \%$ sucrose, and frozen at $-80^{\circ} \mathrm{C}$. Coronal cryostat sections $10 \mu \mathrm{m}$ thick were stained with cresyl violet for Nissl substance. The specimens were evaluated under light microscopy at magnifications of $2.5 \times, 10 \times$, and $40 \times$. Sections were evaluated for neuronal depletion, signs of necrosis, or apoptosis (Lange et al., 1999; Garcia et al., 1978).

Statistical evaluation. Data analysis was performed using Statistica (version 6; StatSoft, Tulsa, OK). A repeated measures ANOVA model was used with "group" as between-subject factor (four levels: ANI-motor cortex, vehicle-motor cortex, ANI-parietal cortex, ANI-cerebellum), "session number" as within-subject factor (eight levels for the reaching performance and 8 or 12 levels for latencies), and "percentage of successful reaches" or "latency between pellet removal and door opening" as dependent variable. Post hoc Tukey's honestly significant difference (HSD) tests were computed for group. Data are provided as mean \pm SEM.

\section{Results}

Experiment 1

In motor cortex, protein synthesis inhibition was $84.0 \pm 1.44 \%$ $60 \mathrm{~min}$ after the first ANI injection. At $24 \mathrm{hr}$, PSI was reduced to $72.0 \pm 4.68 \%$. At $48 \mathrm{hr}$ after the second ANI injection, protein synthesis was normalized (PSI, $6.9 \pm 15.7 \%$ ). In cerebellum and

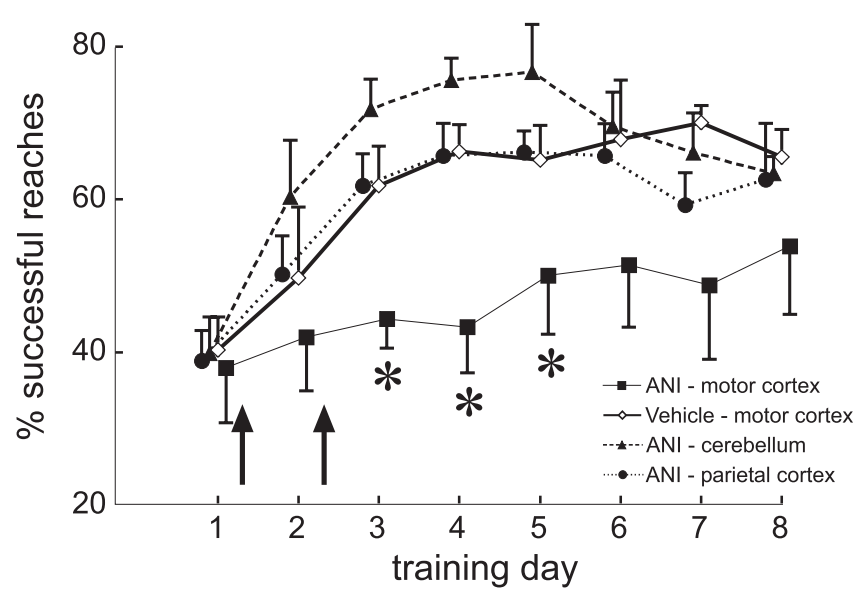

Figure 2. Effects of protein synthesis inhibition on motor skill learning (experiment 3). Inhibition of protein synthesis in motor cortex by local injection of ANI at the time points indicated by arrows impairs motor skill acquisition for $4 \mathrm{~d}$. In contrast, ANI injections into cerebellum or parietal cortex do not affect motor skill learning $\left({ }^{*} p<0.05\right)$. After protein synthesis is restored (day 4), learning resumes but at a lower rate (see Discussion).

parietal cortex, PSI was $87.3 \pm 0.17$ and $85.9 \pm 2.31 \%$ at $60 \mathrm{~min}$, respectively.

\section{Experiment 2}

The spread of India ink in the motor cortex, parietal cortex, and cerebellum was $2.1 \pm 0.1 \times 2.0 \pm 0.2 \mathrm{~mm}^{2}, 1.7 \pm 0.1 \times 1.8 \pm 0.1$ $\mathrm{mm}^{2}$, and $1.9 \pm 0.1 \times 2.6 \pm 0.1 \mathrm{~mm}^{2}$ (vertical times horizontal), respectively (Fig. 1). The vertical spread was limited by the white matter beneath. Hence, the vertical extent of the ink stain in parietal cortex was significantly smaller than in motor cortex $\left(p_{t \text { test }}=0.004\right)$ (parietal cortex is thinner than motor cortex) (Paxinos and Watson, 1998). No difference was found between the cortices for horizontal spreading $\left(p_{t \text { test }}=0.18\right)$. Horizontal spread in cerebellum was significantly larger than horizontal spread in either cortical region $\left(p_{t \text { test }}=0.02\right.$ for motor and $p_{t \text { test }}=0.0003$ for parietal cortex $)$. Vertical spread was not different.

Diffusion of ANI was estimated according to Nicholson (1985): the concentration of ANI at $2.5 \mathrm{~mm}$ distance from the injection point is $98.7 \%$, at $6 \mathrm{~mm} 92.7 \%$ relative to the concentration at the cannula (after $1 \mathrm{hr}$ of diffusion, assuming a free diffusion coefficient of $8.4510^{-6} \mathrm{~cm}^{2} / \mathrm{sec}$ and a tortuosity of 1.6) (C. Nicholson, personal communication).

\section{Experiment 3}

Injection of ANI into motor cortex impaired skill learning (Fig. 2). In comparison with control groups, mean session performance improved only slightly during training sessions 1-4. After session 4, reaching showed clear improvement again. In repeated measures ANOVA, significant effects of group $(F=3.4 ; p=$ $0.036)$ and session $(F=16.48 ; p<0.0001)$ were found. The interaction between group and session was not significant $(F=$ $1.4 ; p=0.115)$. Post hoc Tukey's HSD tests for group showed significant differences between ANI-motor cortex and other groups $(p<0.05)$. Univariate tests per session revealed significant effects of group for sessions $3(F=5.1 ; p=0.008), 4(F=8.3$; $p=0.0007)$, and $5(F=3.9 ; p=0.022)$.

Latencies between pellet removal and subsequent door opening decreased during pretraining (sessions -3 to 0 ) and to a lesser extent during reach training (Fig. 3). If pretraining and training sessions were considered (12 levels of within-subject factor session), the effect of session was significant $(F=30.0 ; p<0.00001)$ but not the effect of group $(F=1.77 ; p=0.19)$ or the interaction 


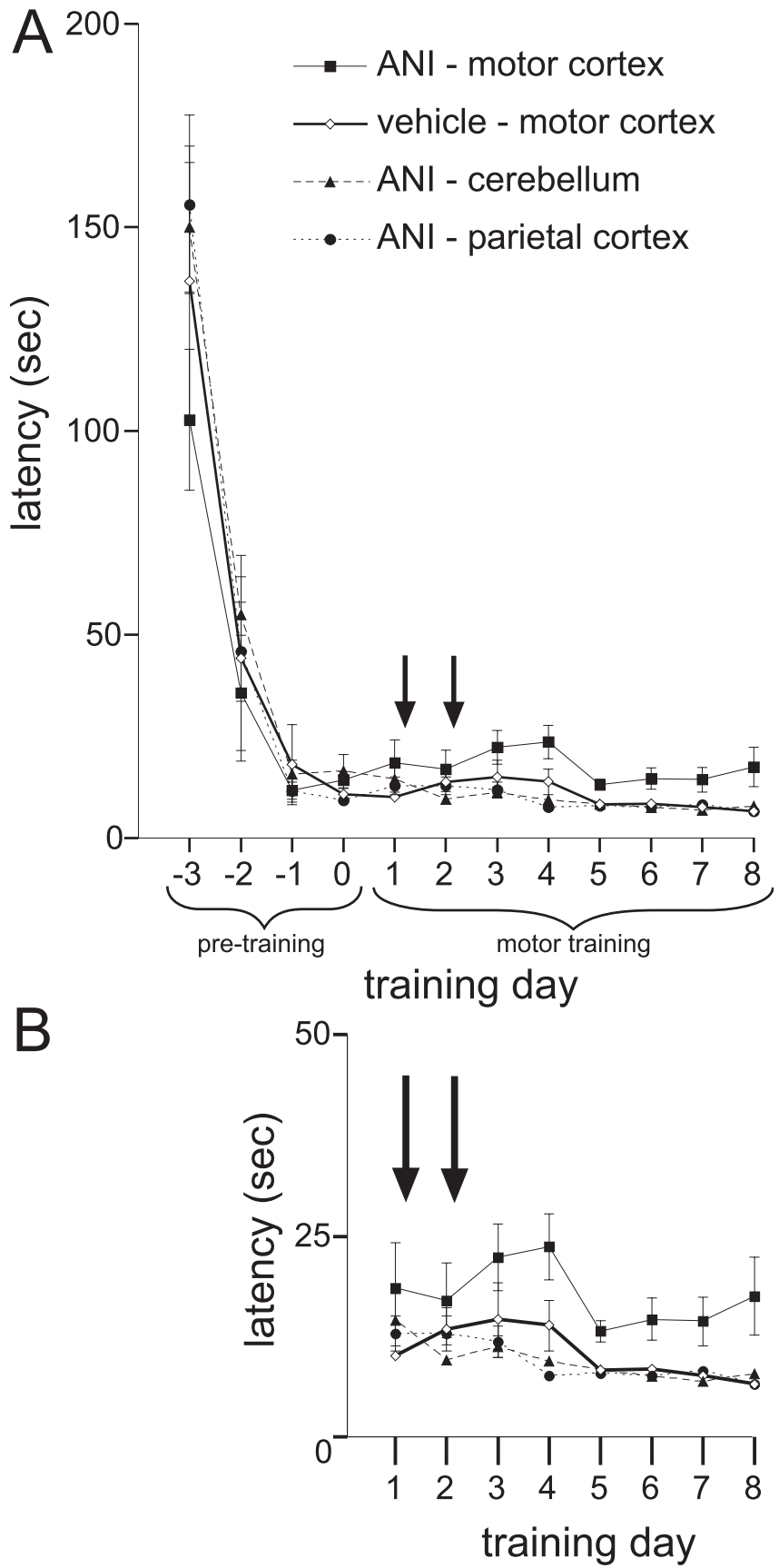

Figure 3. Effects of PSI on latencies between pellet removal and door opening (experiment 3). $A$, During pretraining (sessions $3-0$, pellet accessible by tongue) and subsequent forelimb reach training (sessions 1-8), latencies decrease exponentially. PSI in motor cortex (ANI-motor cortex group) does slightly but nonsignificantly increase latencies. $B$ shows this increase on a stretched $y$-scale. Time points of ANl injections are indicated by arrows.

$(F=0.88 ; p=0.65)$. If only training sessions (i.e., sessions $1-8)$ were included, neither was there a significant effect of session $(F=0.58 ; p=0.77)$ nor of group $(F=0.70 ; p=0.56)$ or the interaction between the two $(F=0.58 ; p=0.93)$. Post hoc Tukey's HSD tests revealed no significant differences for group in either model.

\section{Experiment 4}

Injections of ANI into motor cortex after sessions 11 and 12 did not affect reaching performance (Fig. $4 A$ ). Histological evaluation of motor cortex sections through and adjacent to the cannula
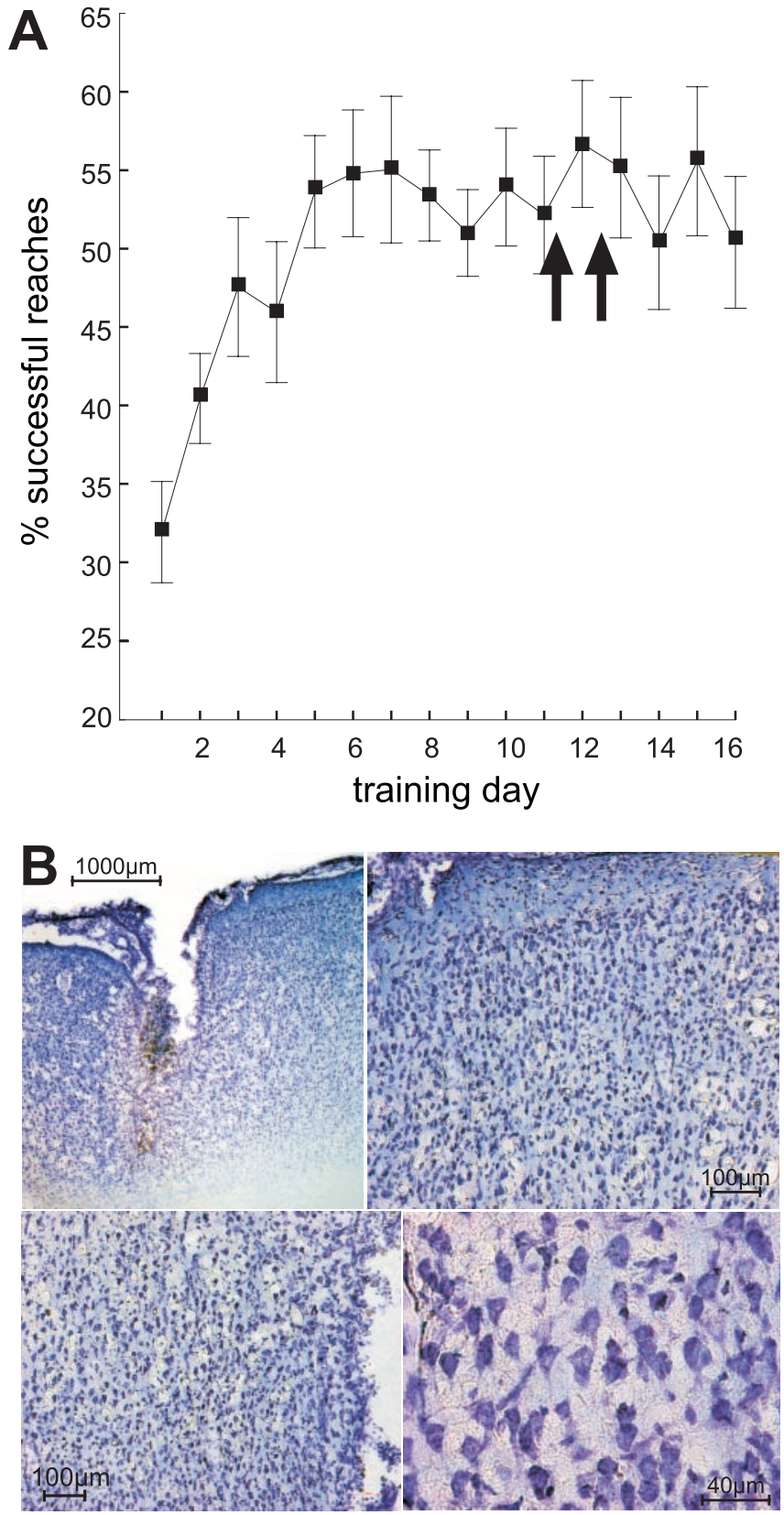

Figure 4. Effects of PSI on motor performance (experiment 4). A, To test whether PSI in motor cortex induces motor deficits, animals were injected with anisomycin during the plateau phase of performance (immediately after sessions 11 and 12). No performance decline is observed. $B$, Motor cortical histology (cresyl violet) obtained from one exemplary animal implanted with the cannula system and injected twice with ANI provides no evidence for cortical injury apart from the needle tract and a small area of hemorrhagic transformation just below the tip of the cannula.

tract did not reveal any signs of neuronal damage apart from the tract itself and a small area of hemorrhagic transformation immediately below the cannula tip in one of the three animals that were evaluated histologically (Fig. $4 B$ ).

\section{Discussion}

The data demonstrate that successful motor skill learning depends on local protein synthesis in motor cortex. Skill learning is impaired if PSI is induced by injection of ANI into the representation of the trained forelimb immediately after daily training. After protein synthesis is restored, learning is reinstated. In con- 
trast, ANI injections into the ipsilateral cerebellar hemisphere do not inhibit motor skill learning. Neither control injections of ANI into parietal cortex nor vehicle injections into motor cortex impaired skill learning. Once the motor skill has developed and a performance plateau is reached, PSI in motor cortex does not affect motor performance.

Protein synthesis is required for successful learning in various paradigms. It was recently shown that local injections of ANI into hippocampus inhibit spatial learning (Naghdi et al., 2003). Learning of instrumental tasks (lever pressing for food) is impaired by ANI injections into the nucleus accumbens (Hernandez et al., 2002). After a single training session, recall of learned content is impaired by PSI (Davis and Squire, 1984). This even occurs if PSI is induced after training, emphasizing a predominant effect of PSI on retention-consolidation as opposed to acquisition (Davis and Squire, 1984).

Our data demonstrate that these effects of PSI can be transferred to motor skill learning and that learning-relevant protein synthesis happens in motor cortex. A closer interpretation of our observations has to consider the ANI application schedule. Drug injection was performed immediately after sessions 1 and 2 . The procedure was performed within $<5$ min of the last reaching movement. By $1 \mathrm{hr}$ after ANI application, we saw $84 \%$ of PSI. Using identical methodology to assess the level of PSI, Rosenblum et al. (1993) found that PSI peaks at $\sim 30$ min postinjection and slowly decreases thereafter. In our setup, PSI was reduced to $72 \%$ by $24 \mathrm{hr}$ and was fully released after $48 \mathrm{hr}$. Maximum levels of PSI measured here were below the threshold of $90 \%$ postulated by Davis and Squire (1984) for PSI to impair memory consolidation, but they were above the $80 \%$ level proposed by others (Quinton and Kramarcy, 1977). This may be related to the timing of the measurement, which may have missed the peak effect at 30 min; more likely, it is a consequence of the large sampling area and thereby inclusion of tissue with low ANI concentration and low protein synthesis inhibition. The tissue volume affected by ANI is determined by: (1) the initial spread of the injected volume of the drug driven by the injection pressure, and (2) subsequent diffusion (Nicholson, 1985). The first point was addressed by the India ink experiment; ink consists of large carbon particles, which do not relevantly diffuse within brain tissue (A. R. Luft and C. Nicholson, personal communication). This experiment demonstrated a spread of $\sim 2 \mathrm{~mm}$ diameter, which is in accordance with values predicted on theoretical grounds (Nicholson, 1985). The estimation of ANI diffusion revealed still high concentrations at a distance of $6 \mathrm{~mm}$ from the injection point. Because of this diffusion, the volume of a relevant ANI effect must be larger than the volume reached by India ink. Because in the tissue block comprising the entire motor or parietal cortex $\left(4 \times 5 \mathrm{~mm}^{2}\right)$ or the cerebellar hemisphere, PSI was slightly below $90 \%$ (the value postulated for PSI effects on learning), the volume affected by ANI should be slightly less than the volume of these tissue blocks. Together, it can be assumed that ANI affected the entire forelimb representations in motor cortex (Neafsey et al., 1986) and cerebellum (Atkins and Apps, 1997).

We have no reason to believe that other effects of ANI, such as inhibition of noradrenergic processes (Freedman et al., 1982), activation of mitogen-activated protein kinases (MAPK) p38 and c-Jun N-terminal protein kinase (JNK) (Hazzalin et al., 1998), and impairment of motor performance (Davis and Squire, 1984), impaired motor learning. Neuromodulatory effects of norepinephrin in cerebellum may be important for motor learning (Bickford, 1995), but no such effects have been reported for motor cortex. In our data, ANI injections into cerebellum did not impair motor learning. Therefore, assuming that noradrenergic inhibition in motor cortex accounts for learning deficits would be far-fetched. Activation of MAPK by ANI is more likely to contribute to neuroplasticity than to impair it (Thomas and Huganir, 2004). Effects of ANI on motor performance were excluded here by showing that ANI had no effect in overtrained animals.

Therefore, ANI can be assumed to have impaired motor learning by inducing PSI in the forelimb representation of motor cortex during the intersession rest period. During this time window, two processes occur. First, skills acquired during the previous training session are consolidated. Skill consolidation may require structural modifications in cortical networks that are mediated by de novo protein synthesis. This process is likely affected by PSI. Second, additional skill improvement occurs without training, possibly by means of a "self-rehearsal" within the motor system (Karni et al., 1998; Walker et al., 2003). In humans, this improvement over rest periods depends on interval sleep (Walker et al., 2003). Our training schedule controlled for effects of sleep, because training was always performed at the beginning of the rat's active phase. However, the present data provide no insights into the relationships of sleep, PSI, and self-rehearsal.

Intersession skill improvements were abolished until training session 4. Between sessions 4 and 5, reaching skills in ANI-motor cortex animals improved (Fig. $2 \mathrm{~A}$ ). At that time, protein synthesis in motor cortex was restored (PSI below 10\%). This demonstrates that the effect of PSI on motor skill learning is in part reversible. But this improvement was smaller than the initial improvement in control animals. By day 8, performance in the ANImotor cortex group was still somewhat lower than in controls (although the difference was not significant). This observation may point to a persistent learning deficit that outlasts PSI. In a recent study, Kleim et al. (2003) suggested that PSI affects motor cortex somatotopy in a persistent manner (at least for $4 \mathrm{~d}$ after ANI injection). Such effects of PSI provide a possible explanation for persistent learning deficits.

The observation that motor learning was not impaired by PSI in cerebellum or parietal cortex does not allow the conclusion that these areas are not involved in motor consolidation processes. They may be, for example, at a different time point during skill acquisition than the times studied here. Because parietal (nonsomatosensory) cortices are less lateralized than motor cortex, bilateral injections would have been required to fully elucidate the role of the parietal cortex in motor learning. Posteriorparietal injections were designed as a control intervention and demonstrate that the effects of PSI on motor learning are specific to motor cortex. The cerebellum has a lateralized organization, although the degree of lateralization may be less than in motor cortex (Luft et al., 1998; Leergaard et al., 2000; Matsushita and Xiong, 2001). However, even with unilateral PSI, some dampening of the learning curve would be expected if protein synthesis in cerebellar cortex is required for skill learning. In contrast, Figure 2 shows a subtle (nonsignificant) elevation of the learning curve of ANI-cerebellum animals.

Latencies between pellet removal and subsequent door opening reflect the learning of conceptual (door opening to get access to the food) and spatial components of the task (where the pellet is in relationship to the cage geometry). They measure a learning process with similarities to instrumental learning (e.g., pressing a lever to obtain food) (Hernandez et al., 2002). Latencies were measured to test whether ANI injections had affected learning components other than motor learning (i.e., conceptual and spatial components). Our data show a subtle and nonsignificant deterioration after unilateral ANI injections into motor cortex 
but not after injections into parietal cortex or cerebellum. We believe that this deterioration reflects a contamination through the impairment of motor learning: animals perform worse in the reaching task and receive less food reward; therefore, they are less "enthusiastic" than controls about opening the door. An alternative interpretation would be that motor cortex protein synthesis has a role in instrumental learning. However, this interpretation seems unjustified, because our study was not designed to test for effects of PSI on instrumental learning.

In summary, this study demonstrates that early acquisition of a motor skill requires de novo protein synthesis in motor cortex after training. PSI induced unilaterally in cerebellum or posterior parietal cortex at identical time points does not interfere with motor learning. Once the task is learned, performance is not affected by PSI in motor cortex. Additional investigations are needed to identify which proteins are synthesized in motor cortex during motor skill learning.

\section{References}

Arshavsky YI (2003) Long-term memory: does it have a structural or chemical basis? Trends Neurosci 26:465-466.

Atkins MJ, Apps R (1997) Somatotopical organisation within the climbing fibre projection to the paramedian lobule and copula pyramidis of the rat cerebellum. J Comp Neurol 389:249-263.

Berman DE, Dudai Y (2001) Memory extinction, learning anew, and learning the new: dissociations in the molecular machinery of learning in cortex. Science 291:2417-2419.

Bickford P (1995) Aging and motor learning: a possible role for norepinephrine in cerebellar plasticity. Rev Neurosci 6:35-46.

Brown IE, Bower JM (2001) Congruence of mossy fiber and climbing fiber tactile projections in the lateral hemispheres of the rat cerebellum. J Comp Neurol 429:59-70.

Davey K, Epstein CM, George MS, Bohning DE (2003) Modeling the effects of electrical conductivity of the head on the induced electric field in the brain during magnetic stimulation. Clin Neurophysiol 114:2204-2209.

Davis HP, Squire LR (1984) Protein synthesis and memory: a review. Psychol Bull 96:518-559.

Freedman LS, Judge ME, Quartermain D (1982) Effects of cycloheximide, a protein synthesis inhibitor, on mouse brain catecholamine biochemistry. Pharmacol Biochem Behav 17:187-191.

Garcia JH, Lossinsky AS, Kauffman FC, Conger KA (1978) Neuronal ischemic injury: light microscopy, ultrastructure and biochemistry. Acta Neuropathol (Berl) 43:85-95.

Gould TJ, Rowe WB, Heman KL, Mesches MH, Young DA, Rose GM, Bickford PC (2002) Effects of hippocampal lesions on patterned motor learning in the rat. Brain Res Bull 58:581-586.

Hallett M (2001) Plasticity of the human motor cortex and recovery from stroke. Brain Res Brain Res Rev 36:169-174.

Hazzalin CA, Le Panse R, Cano E, Mahadevan LC (1998) Anisomycin selectively desensitizes signalling components involved in stress kinase activation and fos and jun induction. Mol Cell Biol 18:1844-1854.
Hernandez PJ, Sadeghian K, Kelley AE (2002) Early consolidation of instrumental learning requires protein synthesis in the nucleus accumbens. Nat Neurosci 5:1327-1331.

Hikosaka O, Nakamura K, Sakai K, Nakahara H (2002) Central mechanisms of motor skill learning. Curr Opin Neurobiol 12:217-222.

Karni A, Meyer G, Rey-Hipolito C, Jezzard P, Adams MM, Turner R, Ungerleider LG (1998) The acquisition of skilled motor performance: fast and slow experience-driven changes in primary motor cortex. Proc Natl Acad Sci USA 95:861-868.

Kleim JA, Bruneau R, Calder K, Pocock D, VandenBerg PM, MacDonald E, Monfils MH, Sutherland RJ, Nader K (2003) Functional organization of adult motor cortex is dependent upon continued protein synthesis. Neuron 40:167-176.

Lange MS, Johnston MV, Tseng EE, Baumgartner WA, Blue ME (1999) Apoptosis detection in brain using low-magnification dark-field microscopy. Exp Neurol 158:254-260.

Leergaard TB, Alloway KD, Mutic JJ, Bjaalie JG (2000) Three-dimensional topography of corticopontine projections from rat barrel cortex: correlations with corticostriatal organization. J Neurosci 20:8474-8484.

Li CS, Padoa-Schioppa C, Bizzi E (2001) Neuronal correlates of motor performance and motor learning in the primary motor cortex of monkeys adapting to an external force field. Neuron 30:593-607.

Luft AR, Skalej M, Stefanou A, Klose U, Voigt K (1998) Comparing motion and imagery related activation in the human cerebellum: a functional MRI study. Hum Brain Mapp 6:105-113.

Matsushita M, Xiong G (2001) Uncrossed and crossed projections from the upper cervical spinal cord to the cerebellar nuclei in the rat, studied by anterograde axonal tracing. J Comp Neurol 432:101-118.

Nader K (2003) Memory traces unbound. Trends Neurosci 26:65-72.

Naghdi N, Majlessi N, Bozorgmehr T (2003) The effects of anisomycin (a protein synthesis inhibitor) on spatial learning and memory in CAl region of rats hippocampus. Behav Brain Res 139:69-73.

Neafsey EJ, Bold EL, Haas G, Hurley-Gius KM, Quirk G, Sievert CF, Terreberry RR (1986) The organization of the rat motor cortex: a microstimulation mapping study. Brain Res 396:77-96.

Nicholson C (1985) Diffusion from an injected volume of a substance in brain tissue with arbitrary volume fraction and tortuosity. Brain Res 333:325-329.

Paxinos G, Watson C (1998) The rat brain in stereotaxic coordinates. New York: Academic.

Quinton EE, Kramarcy NR (1977) Memory impairment correlates closely with cycloheximide dose and degree of inhibition of protein synthesis. Brain Res 131:184-190.

Rosenblum K, Meiri N, Dudai Y (1993) Taste memory: the role of protein synthesis in gustatory cortex. Behav Neural Biol 59:49-56.

Steward O, Schuman EM (2001) Protein synthesis at synaptic sites on dendrites. Annu Rev Neurosci 24:299-325.

Thomas GM, Huganir RL (2004) MAPK cascade signalling and synaptic plasticity. Nat Rev Neurosci 5:173-183.

Walker MP, Brakefield T, Hobson JA, Stickgold R (2003) Dissociable stages of human memory consolidation and reconsolidation. Nature 425:616620 . 